\title{
La lógica de la acción colectiva: tres modelos de análisis de la participación política no institucional
}

\author{
J. F. VALENCIA \\ Universidad del País Vasco

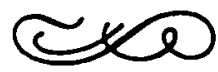 \\ Resumen
}

En el presente estudio se sometieron a prueba tres Modelos explicativos de la Participación Política No Institucional, el Modelo Actitudinal (que incluia Actitud y Norma Social -Fishbein y Ajzen), el Modelo Motivacional de Recursos (que incluia Motivo Selectivo, Social, y Colectivo - Klandermans) y el Modelo Psicosocial (que incluia recursos a diferentes niveles de análisis).

Tomando el Porcentaje de Varianza Explicada como un indice de poder explicativo, el Modelo Psicosocial fue el que mayor peso obtuvo. También se demostró un mayor peso explicativo del Modelo Motivacional de recursos sobre el Actitudinal.

Finalmente, de cara a la Explicación de la Lógica de la Acción Colectiva, se asume la importancia de la intencionalidad de los actores colectivos, pues la explicación causal no es suficiente. En otras palabras, se asume la necesidad de tomar en cuenta que las razones pueden ser causas del comportamiento.

Palabras clave: participación politica

\section{The logic of Collective Action: Three Models on Political Non Institutional Participation}

\begin{abstract}
In the present study were tested three different Models on Political Non Institutional Participation, The Attitudinal Model (including Attitude and Social Norms -Fishbein $\mathcal{E}$ Ajzen), The Motivational Model (including Selective, Social and Collective Motives -Klandermans) and The Psychosocial Model (including resources at the individual, social, and structural-ideological level).

Taking the Explained Variace as an index of Explanatory Power, The Psichosocial Model had the most predictive weight in order to take a decision to participate. It was also showed that The Motivational Model had more explanatory weight than the Attitudinal Model.

Finally, we assume the importance of taking into account the intentionality of collective actors in order to explain The Logic of Collective Action, because causal explanation is not sufficient. We assume the neccesity to take into account that reasons can also be causes of behavior.
\end{abstract}

Key words: Political Participation

Dirección del autor: Departamento de Psicología Social

Facultad de Filosofía y CC Educación, 20014 San Sebastián 


\section{INTRODUCCION}

El significado de la participación política, tanto para el individuo como para el sistema político, proviene de la interacción entre autoridades politicas y ciudadanos, que se da a través de la mediación de las instituciones políticas. En esta interacción un elemento fundamental, en un marco democrático, será el intento por parte de los ciudadanos de influir en las decisiones políticas (Verba y Nie 1972).

Si bien tradicionalmente el estudio de esta relación se había reducido al comportamiento electoral (Kinder y Sears 1985, Sears, 1987), hoy en día se viene aceptando la existencia de diferentes comportamientos en los sistemas democráticos (Milbrath 1981, Barnes y Kaase 1979, Seliktar 1986, Lederer 1986) los cuales abarcan desde los Comportamientos Convencionales, hasta el recurso a técnicas de "acción directa" incluyendo el uso de la violencia como extremo del continuum de la participación No Convencional (Kaase y March 1979b). Ambos tipos de participación, Convencional y No Convencional, anteriormente se entendieron como exclusivos; actualmente sin embargo se asume todo un repertorio de comportamientos políticos bajo el epígrafe de Acción Política (Barnes y Kaase 1979), el cual incluye desde el comportamiento de voto hasta comportamientos revolucionarios (Kinder y Sears 1985, Hermann 1986).

En este sentido puede ser fundamental diferenciar entre legitimidad y legalidad en los diversos comportamientos políticos (Smelser 1963, Muller 1972), pues pone en crisis el concepto de Participación Convencional-No Convencional. Así, mientras la Participación Convencional se redujo al comportamiento electoral, la Participación No Convencional ha sido definida como «aquellos comportamientos que no se corresponden a las normas legales y costumbres que regulan la participación política en un régimen determinado" (Kaase y March 1979a, 41). De cara a una definición operacional del término, pensamos que el término Participación No Convencional, como señala Sabucedo (1984, Sabucedo y Sobral 1986), está sujeto a la oscilación de las condiciones sociales, de manera que lo que resulta no convencional en un momento puede resultar habitual y aceptado poco después.

Parece pues, más adecuado hablar de Participación Política No Institucional (PPNI); que hará referencia al conjunto de comportamientos dirigidos a influir en las decisiones políticas del poder establecido que utilizan çauces no institucionales.

\section{Teorías Explicativas de la Participación Política No Institucional}

Tradicionalmente la explicación de la PPNI se centró en el análisis de factores de corte individual, tales como rasgos de personalidad (Adorno et al. 1950), la marginalidad, anomia, alienación y ansiedad (Lebon 1961, Freud 1923, Kornhauser 1959, Arendt 1963), el descontento o privación relativa (Davis 1959, Davies 1963, Gurr 1970), como factores únicos explicativos de dicha participación. Sin embargo, la investigación empírica no ha aportado evidencia en este sentido (Klandermans 1983, Obershall 1973, Moore 1975, Barnes y Kaase 1979, Klandermans et al. 1987, Dubé y Guimond 1986, Gurney y Tierner 1982, McAdam et al. 1988). 
Las teorias dominantes hasta los años 70 (Collective Behaviour, Sociedad de Masas, Privación Relativa, Escuela Institucional) hacían hincapié en aspectos puramente individuales y en tensiones estructurales de los cambios sociales rápidos, generadores de actores políticos anómicos o descontentos.

El impacto producido por la «explosión de participación" (Almond y Verba 1965,1980 ) de los años $60 / 70$ ha modificado la visión que proyectan tanto los prácticos como los teóricos de la participación. Así la participación en acciones colectivas era vista como a) participación escasa de gente, donde b) los descontentos eran transitorios, c) los comportamientos institucionales y no institucionales eran considerados como diferentes, d) los actores sociales eran a-racionales cuando no i-racionales.

Los movimientos políticos de los años 70 cuestionaron las perspectivas anteriores, provocando con ello un relevo en las corrientes teóricas al uso, de modo que cristalizó de una manera formal la Teoría de los Recursos para la Movilización (TRM) (Obershall 1973, McCarthy y Zald 1973, 1977, Gamson 1975, Tilly 1978).

Esta nueva perspectiva, a diferencia de la anterior, va a centrar su atención en los siguientes aspectos: a) el continuum entre los comportamientos institucionales y no institucionales, b) la racionalidad de los actores en dichos movimientos, $c$ ) el rol de los movimientos sociales como agentes de cambio social.

La TRM propone la participación en movimientos colectivos como un proceso de toma de decisión racional, por medio del cual la gente sopesa los costos y beneficios de su participación.

Igualmente, esta teoría propone «un modelo multifactorializado de la formación de los Movimientos sociales... enfatizando la organización y las oportunidades políticas» (Jenkins 1983, 537). Es decir, enfatiza a) la complejidad de la acción social, b) hace referencia a elementos estructurales como la disponibilidad de recursos para la colectividad y las redes sociales, c) hace finalmente hincapié en la racionalidad de la participación en dichas acciones.

Esta especial atención a la racionalidad del actor colectivo será el resultado de la toma de conciencia de diversos factores en las ciencias sociales: los resultados de la Historia Moderna sobre el descubrimiento de los Prejuicios de clase (Groh 1986), la toma de contacto con la Filosofía Moral Escocesa (Elster 1984), las aplicaciones de la Teoría Económica a los conflictos sociales (Olson 1985, Hirschman 1970), el descubrimiento de que la Ciencia y la Historia no son cosas separadas (Moscovici 1986), así como el hecho de que los cambios estructurales rápidos rompen lazos a nivel micro pero crean nuevos lazos a un nivel superior (Hechter 1975, Nielsen 1980, 1985, Della Porta y Mattina 1986).

Las aportaciones, dicho de una manera general, de esta nueva visión sobre los comportamientos colectivos serán los siguientes: 1 . las oportunidades de acción, 2. las redes sociales, 3 . las organizaciones, 4. la distribución de recursos, 5 . un modelo de actor racional.

\section{La Lógica de la Acción Colectiva}

Una polémica básica que ha aparecido en este modelo de actor racional (sopesador de costos y beneficios) ha sido la contraposición entre la Ra- 


\section{8}

cionalidad Colectiva vs Racionalidad Individual, de modo que la presencia de la una incluye la ausencia de la otra, o dicho en otras palabras la oposición dada entre los Incentivos Selectivos frente a los Incentivos Colectivos que tienden a lograr algún tipo de Bien Común a través de la participación en movimientos colectivos.

A esto es a lo que algunos autores (Hardin 1982a, 1982b, Dawes 1980) se refieren con el concepto de «Dilema Social». Esta polémica se ha puesto de relieve a partir del trabajo de Mancur Olson $(1965,1986)$ cuando en su aplicación a movimientos colectivos afirma la oposición que se da entre la racionalidad del objetivo colectivo, y el autointerés del actor (utilitarista) individual. Para este autor la participación en acciones colectivas no será una consecuencia lógica de la tarea racional dirigida a la maximización de la utilidad individual, sino que la racionalidad grupal está en contradicción con la racionalidad individual. Según él, solamente el empleo de sanciones (Incentivos Selectivos Negativos) o de beneficios selectivos (Incentivos Selectivos Positivos), diferentes e independientes del Bien Colectivo podrán convencer y constreñir a un miembro del grupo a participar. Según este autor toda organización política no podrá existir y realizar su tarea sin confiar en una razón «no política» $(1965,133)$. La participación será un SUBPRODUCTO de los beneficios selectivos. Olson plantea una «LEY FERREA" para la participación, la cual adquiere politicidad en la medida que pierde racionalidad, al igual que recupera su significado racional en la medida que renuncia a su bien colectivo y político.

Algunos autores (Moe 1980, Moscovici 1981) apuntan que Olson no acierta en la solución, si bien vislumbra la incoherencia que muchas veces se ha dado entre las actitudes (convenientes) que un individuo posee de cara un fin colectivo, y las [no] acciones [in] útiles (en el sentido de costos-beneficios) de los modelos de utilidad y valor esperado.

Otros autores (Mattei 1986), objetan que Olson, al primar su utilidad racional, suprime el nexo lógico que conectà la elección de participación por parte de los miembros con los objetivos que definen su pertenencia grupal. La solución a este dilema podría darse atendiendo a las siguientes consideraciones que implican tres niveles de análisis diferentes:

a) Por una parte más que de los Incentivos Selectivos, vendrá dada de la asunción de Incentivos Colectivos, siendo estos los que vinculan la fusión de intereses colectivos y grupales (Jenkins 1983, 537). Así se puede entender la importancia de los Incentivos de Solidaridad y Propósito Moral, basados en relaciones grupales pre-existentes (Gamson et al. 1979, Moe 1980, Zurker y Snow 1981) y las representaciones sociales compartidas por dicho grupos (Villarreal y Valencia 1987).

b) Igualmente habrán de tomarse en cuenta los Incentivos psicosociales, tales como la probabilidad de éxito, la importancia de la propia participación y la expectativa de alta participación (Gamson 1975, Schwartz 1976, Klandermans 1984). Este último elemento ha sido criticado por la complejidad que conlleva (Olivier 1984), por lo que el umbral de participación (Grannovetter 1978, Paez 1984) puede ser considerado elemento psicosocial a tener en cuenta.

c) Finalmente pensamos interesante la asunción de Incentivos Selectivos más psicológicos como el entretenimiento (Tullock 1971), «in- 
greso psíquico" (Silver 1974), o incentivos "blandos" (Muller y Opp 1986).

En términos generales, esta "visión dominante» (Schrager 1985) sobre los movimientos sociales, ha sido sujeta a diferentes críticas: a) su olvido de elementos dinámicos como la privación relativa grupal (Snow, Rochford et al. 1986, Guimond et al. 1983, Tajfel 1984), b) su olvido de aspectos Ideológicos como el radicalismo ante el cambio social (Barnes y Kaase 1979), la alineación política (Muller y Opp 1986). Por otra parte, debido a que fundamentalmente ha sido desarrollada por sociólogos, la crítica más importante, desde la Psicología Social, pueda basarse en su olvido de elementos micro sociales (Snow et al. 1980, Klandermans 1985, Schrager 1985), en especial de la falta de integración de recursos individuales y macro sociales.

Recordemos, que en el plano meta-teórico se ha dado un cambio de cara a la explicación de los fenómenos sociales. A diferencia de la «Visión estándar de la Ciencia», el "Nuevo Paradigma» (Bhaskar 1978, 1983) plantea que los fenómenos sociales son "abiertos", complejos, y están estratificados a diferentes niveles. Estos planteamientos son contemplados también por diversos psicólogos sociales (Newcomb 1951, Secord 1982, 1986, Manicas y Secord 1983) concibiendo la Psicología Social como Mediadora.

Por otra parte, en el plano de nuestra propia ciencia, la escuela psicosociológica de Ginebra (Doise 1976, 1978, 1980, 1983) ha intentado poner de manifiesto esta problemática tratando de superar con ella la dialéctica que se mantiene en nuestra disciplina con respecto a su objeto de estudio.

Pensamos que la articulación de los diferentes recursos que los actores colectivos utilizan de cara a la participación a los diferentes niveles (ideológico-estructurales, grupales, inter-intra individuales) podrán ayudarnos a la formulación de un modelo explicativo integrador de la PPNI desde la TRM, si bien somos conscientes que ningún modelo teórico permitirá captar la realidad en toda su complejidad.

El presente trabajo se va a enmarcar en el campo de la Lógica de la acción colectiva. Esta ha sido tradicionalmente estudiada desde diferentes enfoques. Ya en el marco de la Psicología Social, en el nivel más individual, las aproximaciones de la $T{ }^{a}$ de Valor Esperado a las actitudes se han visto como análisis válidos para esta tarea (Fishbein y Coombs 1974, Fishbein, Thomas y Jaccard 1976, Bowman y Fishbein 1978, Echebarría, Páez y Valencia 1988a). Igualmente en el nivel microsocial, los intentos de analizar los aspectos microsociales de la $T{ }^{a}$ de Recursos para la Movilización utilizando Modelos Motivacionales se han considerado básicos de cara a la superación de la problemática de la racionalidad individual-colectiva (Klandermans 1984, Klandermans et al. 1987, Echebarria, Paez y Valencia 1988b). Sin embargo, una aproximación más amplia desde un marco Psicosociológico puede ayudarnos en esta tarea, en el sentido de que la articulación de los factores macrosociales, intergrupales, e individuales en un modelo integrador más amplio que los anteriores puede aportar elementos de superación de ciertas críticas, y darnos una perspectiva más amplia de cara a la explicación de la Acción Colectiva en general y de la Participación No Institucional en particular.

Será el Objetivo fundamental de este estudio analizar y comparar los modelos actitudinales de Valor Esperado (Fishbein y Ajzen 1975, Ajzen y 
Fishbein 1980), el modelo Microsocial de la T. ${ }^{2}$ de Recursos para la Movilización (Klandermans 1984) y el Modelo Psicosocial propuesto.

Hemos utilizado para ello una muestra azarosa (fundamentalmente compuesta por estudiantes y trabajadores). El estudio lo realizamos en 2 fases, una primera de fiabilidad del pretest, realizado entre febrero y marzo de 1986, con estudiantes, y la segunda fase entre octubre y diciembre de 1986, con estudiantes y trabajadores, habiendo obtenido los resultados que se expondrán a continuación.

\section{METODOLOGIA}

\section{Hipótesis Principales}

De los tres modelos sometidos a prueba, el de actitudes, el de recursos microsociales, y el modelo psicosociológico, este último, al articular los diferentes niveles de análisis e incluir variables de corte no sólo individual, habría de proporcionar el mayor nivel de varianza explicada, permitiendo una mejor explicación de la Lógica de la Acción Colectiva. Igualmente, el modelo de Klandermans habría de obtener mayores niveles explicativos que el actitudinal de Fishbein y Ajzen.

Esperábamos encontrar un campo representacional diferenciado para los sujetos políticamente radicales.

\section{Hipótesis relativas a cada uno de los Modelos}

\section{Hipótesis relativas al Modelo de Fishbein y Ajzen}

La introducción del factor Experiencia Anterior aumentaría la Varianza Explicada, y reduciría la varianza de error.

Con respecto al factor Creencias Normativas Personales o Intención Comportamental Ideal, se esperaba a) por una parte no sería una medida similar a la Intención de participación real, b) que explicaría más que la Norma Subjetiva, y que su introducción en el Modelo añadiría varianza explicada y c) finalmente, que su introducción en el modelo como variable mediadora entre la norma subjetiva y la actitud y la intención de participación real aumentaría el efecto de la Norma Subjetiva y la Actitud en la Intención comportamental, no encontrándose influenciada por la experiencia anterior.

\section{Hipótesis relativas al Modelo de Klandermans}

El Motivo Colectivo explicaría más que el Motivo Social y especialmente que el de Recompensa en contraposición a la hipótesis de Olson.

La introducció del Umbral de Participación en el factor «Expectativas de que la participación ayudará al logro del bien colectivo", explicará un mayor porcentaje de varianza, y menor varianza de error.

\section{Hipótesis relativas al Modelo Psicosociológico}

En el Nivel Macro-ideológico se ha encontrado evidencia teórica y empírica apoyando una relación entre la PPNI y el sexo, la edad, el origen, 
la lengua, la clase social, el radicalismo frente al cambio social y la anomia política. Así, con respecto a las hipótesis de este nivel Macro-ideológico, además de las aportaciones de las Teorías de los Recursos para la Movilización, se han encontrado investigaciones sociológicas (Linz, 1981), y psicosociales (Villarreal, 1987), confirmando estas relaciones. También se han encontrado aportaciones teórico-prácticas confirmando relaciones entre participación política no institucional y origen, sexo, edad, clase social, lengua, radicalismo político, radicalismo lingüístico, aunque a un nivel más parcial (Lambert, 1979, Williams, 1979, Giles y Johnson, 1982, Garmendia et al., 1982, Kourevatis y Dobratz, 1982, Rees et al., 1985, Alzate, 1985, Grenshaw, 1986). En función de ello proponemos las siguientes hipótesis:

- Un mayor nivel de participación no institucional se dará en sujetos de sexo masculino.

- Teniendo en cuenta que la mayoría de los sujetos (80\%) oscilaban entre 20-40 años, el mayor nivel de participación lo encontraríamos en sujetos de edad media.

- Un mayor nivel de participación no institucional lo encontraríamos en sujetos con origen del País Vasco.

- Un mayor nivel de participación se encontraría entre sujetos que conocen euskera, aunque mayor peso obtendría la Intención de Cambio Radical Lingüístico.

- Los sujetos de Clase Social baja adoptarían mayores niveles de participación no institucional.

- Se esperaba un mayor nivel de participación entre los sujetos con Intención Radical de Cambio Social.

- Se esperaba un mayor nivel de participación entre sujetos con mayores puntuaciones en Anomia Política.

En el Nivel intergrupal, se ha encontrado evidencia teórica y empirica apoyando una relación entre la PPNI y la Identidad Social [Vasca], Identidad Nacionalista, Identidad Básica, Privación Grupal, Norma Social y Creencias Normativas Personales. Así, con respecto a las variables intergrupales, además de las aportaciones teórico-practicas de Gurr, Tajfel y colaboradores, sobre privación relativa, se ha encontrado evidencia teóricoempírica confirmando estas relaciones como en identidad social (Giles, Bourish y Taylor, 1977, Garmendia et al., 1982), en identidad nacionalista (Garmendia et al., 1982, Nielsen, 1980, 1985, Alzate, 1985), en identidad básica (Maravall, 1978), Garmendia et al., 1982, Rees et al., 1985), en norma social (Etxebarría, Páez y Valencia, 1988a), en creencias normativas (Schwartz, 1976, Wetsby, 1976). En este sentido se proponen las siguientes hipótesis:

- Se esperaba un mayor nivel de participación en sujetos con Identidad Social Vasca alta.

- Se esperaba un mayor nivel de participación en sujetos con Identidad Nacionalista alta.

- Se esperaba un mayor nivel de participación en sujetos con Identidad Básica alta.

- Se esperaba un mayor nivel de participación en sujetos con un nivel de Privación Relativa Grupal alto. 
- Se esperaba un mayor nivel de participación en sujetos con un nivel de Norma Subjetiva alto.

- Se esperaba un mayor nivel de participación en sujetos con Creencias Normativas Personales altas.

En un Nivel Inter e Intraindividual se ha encontrado evidencia teórica y empírica sobre la relación entre PPNI y el Valor de los comportamientos colectivos, la Instrumentalidad de éstos, el Valor del Motivo Colectivo (independencia y socialismo), la Expectativa de participación positiva, el Valor y la percepción de los costos de participar, el Umbral de Participación, la creencia en un Mundo Injusto, la Atribución de Causalidad, la Organización política, la Participación Política Institucional. Así, con respecto a las variables de nivel inter e intraindividual apuntar las aportaciones de la teoría de actitudes y de los modelos de utilidad y valor esperados, en especial el valor del motivo colectivo y expectativas de participación, el valor de movimientos colectivos e instrumentalidad de ellos, el valor y expectativa de los costos de participación, la experiencia anterior, el umbral de participación y la organización política. Además se ha encontrado evidencia de la relación entre Participación Política No Institucional y Atribución de Causalidad externa (Dumont, 1982, Paez y Echebarría, 1986), así como con Mundo Injusto (Gunter y Wober, 1983, Villarreal, 1987), y con la Participación Política Institucional (Barnes y Kaase, 1979, Muller, 1979, 1982, Milbarth, 1981, Lederer, 1986). En este sentido se proponen las siguientes hipótesis:

- Se esperaba un mayor nivel de participación en sujetos con alta valoración de la participación no institucional.

- Se esperaba un mayor nivel de participación en sujetos con Instrumentalidad alta.

- Se esperaba un mayor nivel de participación en sujetos con valoración alta de la Independencia y el Socialismo.

- Se esperaba un mayor nivel de participación en sujetos con alta Expectativa de que la participación ayudará al logro del bien colectivo.

- Se esperaba un mayor nivel de participación en sujetos con un valor bajo de los costos de la participación.

- Se esperaba un mayor nivel de participación en sujetos con una percepción de los costos de la participación baja.

- Se esperaba un mayor nivel de participación en sujetos con Umbral bajo.

- Se esperaba un mayor nivel de participación en sujetos con una visión del Mundo Injusto.

- Se esperaba un mayor nivel de participación en sujetos con una Atribución de Causalidad Externa.

- Se esperaba un mayor nivel de participación en sujetos con mayor Experiencia Anterior.

- Se esperaba un mayor nivel de participación en sujetos con mayor nivel de organización política.

- Se esperaba un mayor nivel de participación no institucional en sujetos con mayor nivel de Participación Política Institucional. 
Basándonos en la literatura sobre el tema de relatos biográficos con militantes politicos de mediados de los 70, hemos construido una Escala de Participación No Institucional, que incluye desde no hacer nada, firmar escritos de presión, participar en manifestaciones, participar en boicots, participar en huelgas, participar en ocupación de edificios, participar en bloqueo de tráfico, hasta participar en enfrentamientos «duros» con la polícía.

Se preguntó a los sujetos por el grado de acuerdo con realizar cada uno de los comportamientos en el futuro de cara al cambio sociopolítico que tendiera hacia la Autodeterminación y la Independencia (npgind) por una parte, y también hacia una sociedad Socialista e Igualitaria (npgsoc). El nivel de Participación No Institucional fue considerado el sumatorio de las puntuaciones en ambas (npgtot).

En el pretest encontramos un coeficiente Alfa de 0,78 para la primera y 0,85 para la segunda, observándose una correlación alta entre ambas $(\mathrm{r}=.80, \mathrm{p}=.001)$.

De cara al análisis de correspondencias calculamos un Potencial de Partipación No Institucional con el sumatorio de la multiplicación de la puntuación en cada uno de los comportamientos por 1 si no había realizado dicho comportamiento en el pasado, y por 2 si lo había realizado.

\section{Medidas de las Variables Independientes}

1. Se preguntó a los sujetos el SEXO $(1=$ masc, $2=$ fem $)$, EDAD, PROVINCIA DE NACIMIENTO Propio y de los Padres ( $1=$ País Vasco, 2 = Estado, 3 = Extranjero).

2. Se preguntó a los sujetos, de cara a la CLASE SOCIAL, la profesión Propia y de su Padre, computando con las normas de FOESSA, y adscribiendo a los estudiantes la de su padre. La categorización de las diversas profesiones se hizo de la forma siguiente: 1 = peonaje y clase baja; 2 = clase media baja, 3 = clase media-media; no encontrándose en la muestra sujetos de categorías superiores.

3. El Conocimiento del EUSKERA; se evaluó con un ítem de 4 respuestas, nulo-bueno.

4. El RADICALISMO LINGÜISTICO; preguntamos qué lengua le gustaría que se hablase en el futuro en el País Vasco, Castellano/Euskera, con 5 respuestas de sólo en castellano a sólo en euskera.

5. La ANOMIA POLITICA; se utilizaron los 3 ítems que centrados en una visión distante de las instituciones sociopolíticas, habían sido validados y fiabilizados por Páez y Echebarría (1986). En el pretest se obtuvo un coeficiente alfa de 0,70. En la investigación produjeron un solo factor que saturó el $69,1 \%$ de la varianza, observando una fiabilidad de 0,75 por el método de las dos mitades.

6. El RADICALISMO SOCIOPOLITICO; tomamos la pregunta sobre Orientación de protesta y cambio social de Inglehart (1984) con 3 respuestas: cambio revolucionario, reformista, conservador.

7. IDENTIDAD SOCIAL VASCA; siguiendo a Garmendia et al (1982) utilizamos el sumatorio de «Vasquismo subjetivo» $\mathrm{y}$ «Vasquismo general». El Vasquismo General constaba de 5 respuestas desde usted se considera Español hasta usted se considera Vasco. El Vasquismo Subjetivo, si 


\section{4}

usted personalmente se considera vasco, con tres respuestas, sí, más o menos, no.

8. IDENTIDAD NACIONALISTA; tomamos la pregunta de FOESSA sobre si usted se considera partidario del centralismo o la independencia, con 4 respuestas eliminando la relativa al federalismo pues en el pretest sólo obtuvo un $2 \%$.

9. IDENTIDAD BASICA; se utilizó la pregunta clásica de con cuál de los siguientes grupos políticos se identifica, extrema derecha-extrema izquierda, con cinco posibilidades de respuesta.

10. PRIVACION RELATIVA; basándonos en los trabajos de Guimond et al (1983) utilizamos para la escala, los items de privación económica («los vascos somos discriminados económicamente por los dirigentes del Estado Español»), privación política («los intereses políticos de los vascos son sistemáticamente olvidados por los dirigentes del Estado Español»), privación político-emocional ( «con la autonomía han desaparecido mis posibles sentimientos de opresión»), y privación lingüistica ("nos encontramos frente al peligro que desaparezca la lengua vasca»). Fueron medidas por ítems de formato Likert de 6 puntos (muy de acuerdo-muy en desacuerdo). La escala obtuvo en el pretest un coeficiente alfa de 0,79 , obteniendo en el trabajo una fiabilidad de .80 por el método de dos mitades, $y$ en el análisis factorial, un solo factor que explicó el $70 \%$ de la varianza.

11. NORMA SOCIAL; tomando como referente saliente la familia y los amigos preguntamos cómo ve su familia su participación y no participación en movimientos colectivos, con formato Likert de 6 puntos, muy de acuerdo-muy en desacuerdo.

12. La MOTIVACION A CONFORMARSE A LA NORMA; se preguntó por la importancia que concede a la reacción de las personas que son importantes para usted, de cara a su participación, con ítem de respuesta formato Likert, 6 puntos, muy de acuerdo-muy en desacuerdo.

La NORMA SUBJETIVA la obtuvimos de la multiplicación de Norma Social y Motivación a Conformarse a la norma.

13. CREENCIAS NORMATIVAS PERSONALES; siguiendo a Ajzen y Fishbein (1973) y a Budd et al (1984) se preguntó a los sujetos si "personalmente pienso que debería participar en movimientos colectivos", con formato Likert de 6 puntos.

14. VALOR DEL CAMBIO SOCIAL; se preguntó a los sujetos en relación al derecho de Autodeterminación e Independencia/una sociedad Socialista e igualitaria, los dos ítems con formato Likert.

15. EXPECTATIVA DE QUE LA PARTICIPACION AYUDARA AL LOGRO DEL BIEN COLECTIVO; siguiendo a Klandermans (1984) utilizamos 3 preguntas: importancia de la propia participación, si mucha gente participase obligaría al Gobierno Central a acceder a los cambios, cuánta gente espera que participe en manifestaciones radicales e ilegales.

El MOTIVO COLECTIVO lo obtuvimos por medio de la multiplicación de Valor del Cambio Social y Expectativa.

16. VALOR DE LOS MOVIMIENTOS COLECTIVOS; preguntamos si está a favor o en contra en relación a la participación en Movimientos colectivos (esto es, tomar parte en manifestaciones, etc.), formato Likert, 6 puntos, a favor - en contra.

17. INSTRUMENTALIDAD; se preguntó si usted cree que los Mo- 
vimientos Colectivos son un elemento facilitador para la consecución de a) la Autodeterminación y la Idependencia, y b) una sociedad Socialista e Igualitaria. Formato Likert. LA ACTITUD se obtuvo de' la multiplicación del valor y la instrumentalidad.

18. VALOR DE LOS COSTOS; se preguntó «qué valor da usted a los Costos (como tiempo, dinero, molestias, represalias, etc.), de participar en manifestaciones radicales e ilegales". COSTOS PERCIBIDOS; se preguntó por «cuáles serían los costos (en tiempo, dinero, molestias, represalias, etc.), si participase en manifestaciones radicales e ilegales".

Si bien en la literatura observamos medidas de beneficios, beneficios y costos, y de costos solamente para operacionalizar el «motivo de recompensa" nosotros nos hemos adherido a la última por ser la utilizada por Klandermans $(1984,509)$.

El MOTIVO DE RECOMPENSA se obtuvo multiplicando los costos percibidos por el valor.

19. ATRIBUCION DE CAUSALIDAD; tomamos los 3 ítems más significativos de la versión revisada por Dumont (1982) de los ítems de atribución sobre problemas sociales de Portes (1971). El pretest observó un coeficiente alfa de 0,72. En el trabajo se obtuvo una fiabilidad de 0,59 por dos mitades, y un solo factor que saturaba el $57 \%$ de la varianza.

20. MUNDO JUSTO-INJUSTO; basándonos en la Creencia de Mundo Justo-Injusto de Lerner (1970) y Lerner y Miller (1978), que si bien ha sido utilizada como rasgo de personalidad por Rubin y Peplau (1973) - con 16 ítems - y por Rubin y Peplau (1975) — 20 ítems-, siguiendo a Dumont (1982) nosotros la hemos considerado como variable motivacional que no puede deslindarse del contexto sociopolítico, más que como rasgo. Hemos tomado los 5 ítems políticos de la 2." versión.

Obtuvieron un coeficiente alfa de 0,65 en el pretest. En el trabajo obtuvieron una fiabilidad de 0,44 por el método de dos mitades, y 2 factores que saturaban el $49 \%$ de la varianza. La problemática de la no unidimensionalidad coincide con recientes trabajos sobre la escala (Hyland y Lann, 1987).

21. EXPERIENCIA ANTERIOR; utilizamos la escala de Inglehart (1979) mejorándola con ítems de participación no institucional, la escala utilizó 11 preguntas con 4 respuestas, de muy a menudo-nunca.

22. INTENCION DE VOTO; se preguntó a qué partido votaría en las próximas elecciones.

23. UMBRAL; preguntamos por el porcentaje mínimo de participantes que necesitaba para incorporarse a una movilización colectiva en una situación general.

24. ORGANIZACION POLITICA; preguntamos si participa como militante o simpatizante con algún partido político, con 4 respuestas de muy amenudo-nunca.

25. INTENCION DE PARTICIPACION INSTITUCIONAL; en función de la literatura y relatos biográficos construimos una escala con: no hacer nada, votar, votar y asistir a actos electorales, hacer campañas, participar en listas electorales, ser representante político en las instituciones vascas, ser representante político en las instituciones del Estado.

Se preguntó a los sujetos por el grado de acuerdo con realizar cada uno de los comportamientos en el futuro de cara al cambio sociopolítico que 
tendiera hacia la Autodeterminación y la Independencia (indppi) por una parte, y hacia una sociedad Socialista e Igualitaria (socppi) por otra. El nivel de Participación Institucional fue considerado el sumatorio de las puntuaciones en ambas (ppitot).

En el pretest encontramos una fiabilidad Alfa de 0,81 para la primera y 0,83 para la segunda, observándose una correlación alta entre ambas $(\mathrm{r}=.70, \mathrm{p} \leqslant .001)$.

\section{RESULTADOS}

\section{Modelo de Fishbein y Ajzen}

Para probar las hipótesis correspondientes a este modelo realizamos, inicialmente, un análisis de Regresión Múltiple por el método stepwise, ordenando que introdujera jerárquicamente, en primer lugar la actitud y la norma social, y que en segundo lugar añadiera el factor experiencia anterior (tabla I). Posteriormente realizamos otro análisis de regresión a fin de encontrar los efectos y la significatividad de cada factor (tabla II). Tomamos como Variable Dependiente la Intención de Participación No Institucional. Los resultados fueron los siguientes:

TABLA I

V.D. Participación no institucional (npgtot)

\begin{tabular}{llllc}
\hline & $\mathbf{R}$ & $\mathbf{r}^{2}$ & $\mathbf{e}$ & $\begin{array}{c}\text { g.l. } \\
\text { (residuales) }\end{array}$ \\
\hline Norma soc. y actitud....................... & .69 & .47 & $.19,21$ & \\
Norma soc., act. y experiencia.......... & .70 & .50 & $.18,7$ & 267 \\
\hline
\end{tabular}

TABLA II

V.D. Participación no institucional

\begin{tabular}{|c|c|c|c|c|c|}
\hline & $\mathbf{R}$ & $\mathbf{r}^{2}$ & $\mathbf{b}$ & $\mathbf{t}$ & $\mathrm{p}$ \\
\hline Actitud......... & .69 & .48 & .58 & 12.08 & .0000 \\
\hline 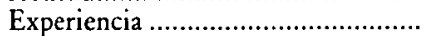 & .70 & .49 & .14 & 2.76 & .0004 \\
\hline Norma subj...................................... & .71 & .51 & .13 & 2.74 & .0064 \\
\hline
\end{tabular}

Figura 1

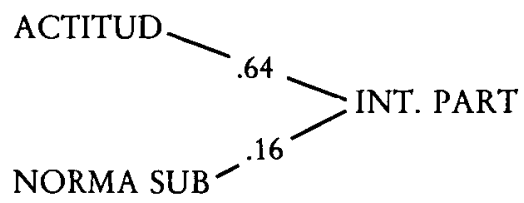

FIGURA 2

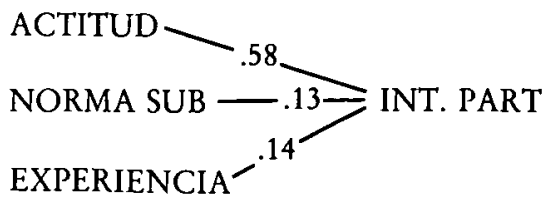

Se observa, con respecto al modelo de Fishbein que la actitud tiene un efecto mayor que la norma subjetiva (Fig. 1), y que la introducción de la experiencia anterior añade $3 \%$ de varianza explicada y reduce $1 \%$ de error (tabla I). El efecto de la experiencia anterior fue mayor que la norma social 
(tabla II, Fig. 2) debido a que el análisis introdujo en segundo lugar la experiencia, con b y p ligeramente mayores. Realizada la Prueba de la Variable Añadida de V.I (Tabachnick \& Fidell, 1984), encontramos una F (1, $267)=15.97 p \leqslant .001$, confirmando por tanto el incremento de la varianza explicada al introducir la experiencia anterior.

Por lo que respecta a la problemática en torno a si la Creencia Normativa Personal (CNP) y la Intención de Comportamiento son dos indicadores del mismo constructo, o por el contrario, pueden considerarse dos variables independientes entre sí, sometimos ambas al procedimiento señalado por Zeller y Carmines (1984), encontrando significativa la diferencia $(\mathrm{p}=.05)$.

Este resultado sugiere que la Creencia Normativa Personal y la Intención de Participación No Institucional, en contra de lo que defienden Fishbein y Ajzen (1973), son dos indicadores diferentes, como sostenían Bentler y Speckart $(1979,1981)$, y Budd et al (1984b). Según Budd et al (1984b, 1985), las Creencias Normativas Personales, o utilizando su terminología, las Intenciones Comportamentales Ideales (ICI) se formarían como resultado de la evaluación de la acción y su percepción de las expectativas de los otros (actitud y norma subjetiva); la Intención comportamental real, en cambio, se basaría en las ICI y en el comportamiento anterior. Estos autores se basan en el hecho de que la gente recuerda su pasado cuando debe tomar una determinación comportamental. Por ello se espera que existan efectos significativos directos e indirectos a través de ICI en la Intención real, mientras la experiencia sólo mostrará efectos directos, sin incidir, en la ICI (Fig. 3).

Con el fin de contrastar dicha hipótesis sometimos los datos a análisis de Regresión múltiple con los siguientes resultados:

TABLA III

\begin{tabular}{lllll}
\hline & $\mathbf{R}$ & $\mathrm{r}^{2}$ & $\mathrm{e}$ & $\begin{array}{c}\text { g.l. } \\
\text { (residuales) }\end{array}$ \\
\hline Norma soc., act. y experiencia .......... & .70 & .50 & $.18,7$ & \\
Norma soc., act., experiencia e ICI .. & .72 & .52 & .18 & 266 \\
\hline
\end{tabular}

TABLA IV

\begin{tabular}{|c|c|c|c|c|c|}
\hline & $\mathbf{R}$ & $r^{2}$ & b & $\mathbf{t}$ & $\mathbf{p}$ \\
\hline Actitud & .68 & .47 & .44 & 7.5 & .0000 \\
\hline ICI & .71 & .51 & .26 & 4.33 & .0000 \\
\hline Experiencia & .72 & .52 & .13 & 2.91 & .0043 \\
\hline 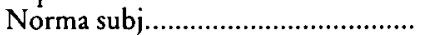 & & & .08 & 1.79 & .0756 \\
\hline
\end{tabular}

Los resultados que se aprecian en la tabla III sugieren, en primer lugar, que la inclusión en el modelo reformulado por Bentler y colaboradores de la ICI incrementa significativamente la varianza explicada por el Modelo $\left(r^{2}=2 \%, f(1,266)=11.11, p \leqslant .001\right)$. En segundo lugar el hecho que la norma subjetiva no entre en la ecuación, si bien es tendencialmente significativa (tabla IV), puede interpretarse en el sentido que su influencia es fun- 
damentalmente indirecta, actuando a través de la intención, más que directamente.

De cara a la hipótesis de la Intención Comportamental Ideal como variable mediadora realizamos un diagrama de senderos (ver Fig. 3) después de haber realizado una regresión múltiple con ICI como variable dependiente con los siguientes resultados:

TABLA V

\begin{tabular}{|c|c|c|c|c|c|}
\hline & $\mathbf{R}$ & $\mathbf{r}^{2}$ & b & $\mathrm{t}$ & $\mathbf{p}$ \\
\hline 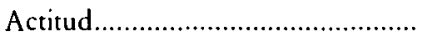 & .70 & .49 & .62 & 14.71 & .0000 \\
\hline 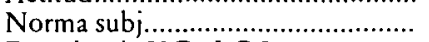 & .73 & .53 & .23 & 5.32 & .0000 \\
\hline Experiencia V.D. I.C.I......................... & \multicolumn{2}{|c|}{ no entra } & & & (ns) \\
\hline
\end{tabular}

TABLA VI

\begin{tabular}{|c|c|c|c|}
\hline & $\begin{array}{l}\text { Efecto } \\
\text { directo }\end{array}$ & $\begin{array}{l}\text { Efecto } \\
\text { indirecto }\end{array}$ & $\begin{array}{c}\text { Efecto } \\
\text { total }\end{array}$ \\
\hline Actitud & .44 & .16 & 60 \\
\hline Norma subi & .08 & .06 & .14 \\
\hline ICI & .26 & & .26 \\
\hline 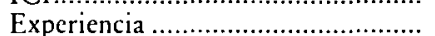 & .13 & & .13 \\
\hline
\end{tabular}

FIGURA 3

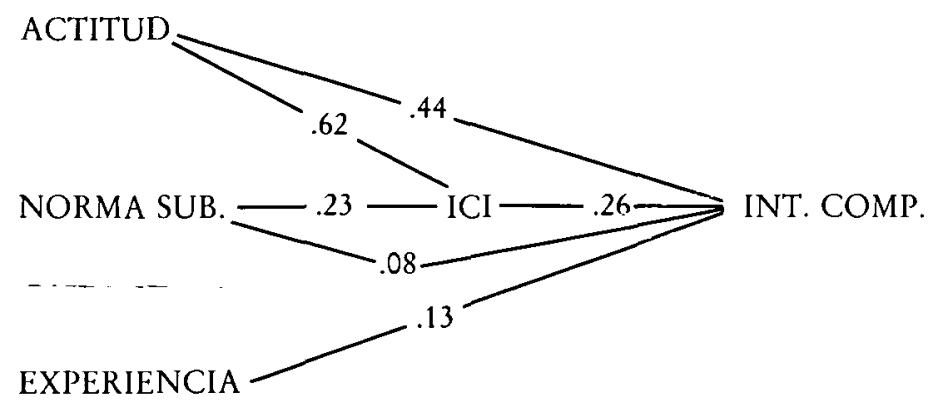

Los resultados sugieren (tabla $\mathrm{V}$ ) que no es a través de ICI como la experiencia tiene incidencia en la intención de participación, confirmando las hipótesis de Bentler y Speckart (1979) y Budd et al. (1984b). Por otra parte se observa que (tabla VI) por medio de los caminos indirectos se aumenta el efecto tanto de la Actitud como de la Norma Subjetiva sobre la intención comportamental.

Resumiendo, estos resultados sugieren, por una parte, que con la muestra utilizada se verifican las hipótesis de la experiencia anterior, pues tiene peso beta ligeramente más alto que la norma subjetiva, el análisis la introduce en segundo lugar antes que la Norma Subjetiva (tabla IV), y agrega significativamente varianza explicada (tabla I). Por otra parte, los resultados sugieren que la ICI puede ser utilizado como factor suplantador de la 
norma social por una parte, o como variable intermedia entre la norma y la actitud y la intención de comportamiento real por otra (tabla V y Fig. 3).

\section{El Modelo de Klandermans}

Recordemos las similutudes señaladas entre el Modelo de Klandermans y el Modelo de Fishbein y Ajzen. El Modelo de Klandermans por una parte, retoma la operacionalición del concepto de Actitud de la Teoría del Valor Esperado al definir el Motivo Colectivo, y por otra, el concepto de Norma Subjetiva (expectativas sobre las respuestas de los otros significativos a la participación y no participación) al operacionalizar el Motivo Social. Una diferencia entre ambos modelos radica en la introducción del Motivo de Recompensa, es decir, el concepto de "incentivo selectivo" de Olson (1965). Otra de las diferencias entre estos dos modelos radicará en el factor «expectativa de participación", en el que Klandermans introducirá elementos de solidaridad grupal como el número esperado de participantes, expectativa de éxito si mucha gente participa, e importancia de la propia participación.

De cara a contrastar las hipótesis relativas al modelo, realizamos un análisis de regresión múltiple stepwise, introduciendo en primer lugar el Motivo Colectivo, y añadiéndole sucesivamente el Motivo Social y el Motivo de Recompensa (tabla VII). Los resultados fueron los siguientes:

TABLA VII

\begin{tabular}{|c|c|c|c|c|c|}
\hline & $\mathbf{R}$ & $r^{2}$ & b & $\mathbf{t}$ & p \\
\hline Motivo colectivo .................................. & .70 & .49 & .65 & 14.36 & .0000 \\
\hline 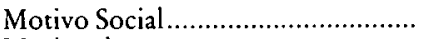 & .71 & .51 & .14 & 3.16 & .0020 \\
\hline Motivo de recompensa ....................... & \multicolumn{2}{|c|}{ no entra } & .07 & -1.75 & .0814 \\
\hline
\end{tabular}

Se observa que el Motivo de Recompensa no juega un papel significativo, siendo su peso beta sólo tendencial, y no añadiendo varianza explicada (tabla VII).

La varianza explicada por el modelo (51\%) es ligeramente superior a la explicada por el modelo original de Ajzen y Fishbein (47\%) (comparar tabla VII y tabla I), y ligeramente superior al modelo reformulado al introducir la experiencia anterior (tabla I).

Globalmente, de cara a la racionalidad de la Acción Colectiva, los resultados sugieren en contra de la hipótesis de Olson que no son los «beneficios selectivos» los importantes para que el «beneficiario franco» participe, sino que en este caso el Valor de un "beneficio colectivo» es mucho más importante de cara a la participación.

Con respecto a la segunda hipótesis, es decir, que la introducción del Umbral en el factor «Expectativa», como elemento que a través del proceso de socialización y la práctica anterior era una base de sentimiento de Solidaridad de cara al comportamiento colectivo, los resultados fueron los de la tabla VIII.

En conclusión podemos extraer a) la introducción del Umbral en el factor Expectativa aumenta la varianza explicada del factor en un $3 \%$ (ta- 


\section{0}

TABLA VIII

V.D. PPNI (npgtot)

\begin{tabular}{|c|c|c|c|c|c|}
\hline & $\mathbf{R}$ & $r^{2}$ & b & $t$ & $\mathrm{p}$ \\
\hline Expect. si muchos part........................ & .51 & .26 & .37 & 6.7 & .0000 \\
\hline Import. propia part......................... & .56 & .32 & .22 & 4.22 & .0000 \\
\hline Umbral & .59 & .35 & -.21 & -4.66 & .0001 \\
\hline 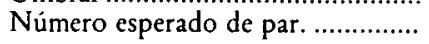 & \multicolumn{2}{|c|}{ no entra } & .05 & 1.01 & $.3100 *$ \\
\hline
\end{tabular}

bla VIII), b) que podría ser utilizado como factor de solidaridad en lugar del número esperado de sujetos.

A modo de resumen, los resultados sugieren: 1. de cara a la comparación de ambos modelos que la diferencia entre el modelo de actitudes y el microsocial estriba en la aportación del factor motivo de recompensa, pero especialmente en el factor Expectativa que la participación ayudará al logro del bien Colectivo. Será este factor el que aumente la varianza del modelo de Klandermans. 2. Por otra parte, y de cara a la racionalidad colectiva frente a la tesis de Olson, es conveniente apuntar que la asunción de factores de solidaridad (expectativa con sus 3 factores) será uno de los elementos que incidan en el individuo a la hora de dejar de hacer el "viaje gratis" y de tomar la decisión de participar. Será éste el elemento diferenciador entre los modelos de racionalidad pública frente a los modelos de racionalidad privada (basados en Utilidad Esperada).

\section{El Modelo Psicosocial}

Con el fin de verificar las hipótesis realizamos un análisis discriminante con la Intención de Participación no Institucional y las Variables sometidas a prueba. Dicho análisis se realizó en función de 3 grupos de la variable dependiente (bajos, medios, altos) tomando para ello las puntuaciones terciles. Presentamos a continuación la tabla de medias y significaciones (tabla IX), la tabla de correlaciones de cada variable en la primera función (tabla X), y la posición de los tres grupos en el eje de coordenadas (Fig. 4).

El análisis clasificó correctamente el $98,08 \%$ de los sujetos, e imponiendo al análisis una función, ésta obtuvo una $X^{2}=508.975, p \leqslant .0000$, con 56 gl., explicando el $98,46 \%$ de la varianza.

Respecto a las variables macrosociológicas los resultados sugieren (tabla IX) que no se verifican las hipótesis correspondientes a sexo, edad y origen. Si en cambio las relativas a la clase social, y euskera.

Las hipótesis relativas a las variables ideológicas quedan confirmadas en el sentido que hay diferencia significativa entre los niveles de participación y una visión distante del sistema político (anomia), radicalismo ideológico, y radicalismo lingüístico.

Las hipótesis relativas a las variables intergrupales, igualmente quedan confirmadas, en el sentido que son los sujetos con alta Identidad Social Vasca, alta Identidad Nacionalista, alta Identidad Básica radical, alta privación relativa grupal, y alta norma social familiar (si bien en esta última tanto los sujetos de participación media como alta, puntúan parecido $(7,73 / 7,70)$ siendo ligeramente inferior la de los últimos $(x=7.50)$. 


\section{1}

TABLA IX

\begin{tabular}{|c|c|c|c|c|c|}
\hline Variable & 1 & 2 & 3 & t & $s$ \\
\hline 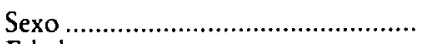 & 1.37 & 1.46 & 1.46 & .80 & $.45^{*}$ \\
\hline Edad ......... & 27.32 & 27.95 & 29.55 & 1.81 & $.16 \%$ \\
\hline 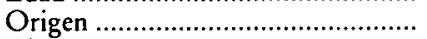 & 2.07 & 2.23 & 1.75 & 1.81 & $.16^{*}$ \\
\hline Clase & 1.78 & 1.53 & 1.46 & 4.32 & .0144 \\
\hline Euskera .......... & 2.35 & 2.63 & 2.95 & 8.33 & .0003 \\
\hline 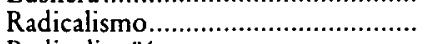 & 1.96 & 2.15 & 2.53 & 35.51 & .0000 \\
\hline 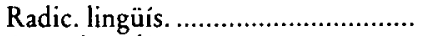 & 3.58 & 3.94 & 4.33 & 18.79 & .0000 \\
\hline 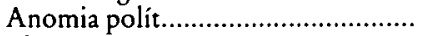 & 9.47 & 10.24 & 13.49 & 41.45 & .0000 \\
\hline 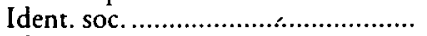 & 6.58 & 7.01 & 7.62 & 13.33 & .0000 \\
\hline 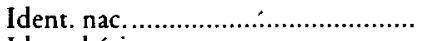 & 2.90 & 3.36 & 3.72 & 32.91 & .0000 \\
\hline 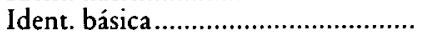 & 3.72 & 3.97 & 4.50 & 35.82 & .0000 \\
\hline Priva. grup. .................................... & 15.32 & 16.97 & 19.97 & 28.55 & .0000 \\
\hline Norma subj.......................................... & 7.28 & 7.73 & 7.70 & 4.75 & .0094 \\
\hline 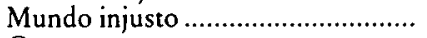 & 22.42 & 23.84 & 26.55 & 20.66 & .0000 \\
\hline Creen. nor. per. ............................ & 3.47 & 4.31 & 5.17 & 60.36 & .0000 \\
\hline Valor m.c. & 3.48 & 4.42 & 5.33 & 62.16 & .0000 \\
\hline Instrumént. & 6.47 & 8.13 & 9.89 & 51.30 & .0000 \\
\hline Valor b.c. & 8.32 & 9.47 & 11.20 & 47.15 & .0000 \\
\hline Expect. b.c. & 8.71 & 10.23 & 12.09 & 37.76 & .0000 \\
\hline Expec. costos & 6.46 & 5.88 & 5.78 & 2.29 & $.0920 \%$ \\
\hline 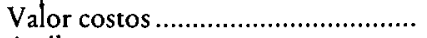 & 6.82 & 6.33 & 6.65 & 1.19 & $.3000 *$ \\
\hline 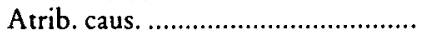 & 12.67 & 14.47 & 16.28 & 38.07 & .0000 \\
\hline 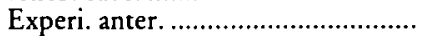 & 18.48 & 21.21 & 24.63 & 18.56 & .0000 \\
\hline 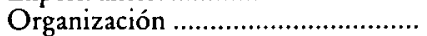 & 1.77 & 2.05 & 2.55 & 10.27 & .0001 \\
\hline 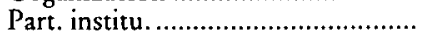 & 14.37 & 25.05 & 30.43 & 12.38 & .0000 \\
\hline
\end{tabular}

Tabla XIII, Var. Dep. Intención de Part. No Inst. (npgrot).

" no significativo: $"$ tendencial.

TABLA X

Función I

Valor mov, colectivos......................................................

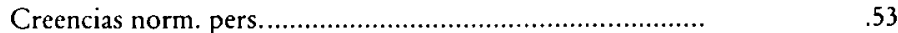

Instrumentalidad ………………………………...................

Valor bien colectivo.........................................................

Expectativa ................................................................. 48

Anomia................................................................

Atribución ........................................................................

Identidad básica................................................................

Identidad nacionalista ...........................................................

Privación relativa ................................................................... 43

Radicalismo ideológico ................................................................

Radicalismo lingǘstico ........................................................

Mundo injusto ..................................................................

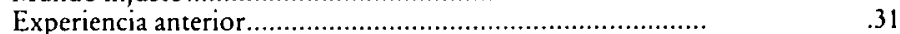

Identidad social ...................................................................

Norma social .....................................................................

Particip. institucional ...............................................................

Organización política ...........................................................

Euskera.............................................................

Clase social ..................................................................

Costos percibidos .................................................................

Valor de los costos ...........................................................

Origen .....................................................................

Sexo

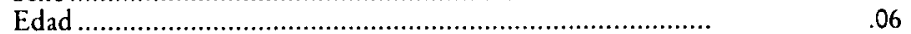




\section{2}

FIGURA 4

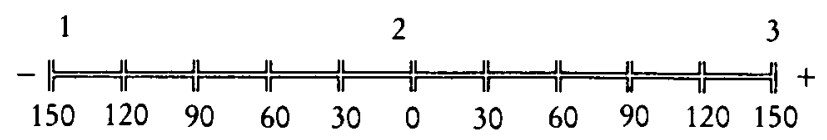

En relación con las variables inter-intraindividuales se verifican las hipótesis propuestas. No se confirmó la hipótesis referida a los costos de la participación; si bien se observa una tendencia en el sentido de que son los que manifiestan una intención de participación radical los que tienen expectativas de costos más bajas, las diferencias no son significativas. No se observan diferencias en el valor atribuido a los costos. Todo ello indica que la percepción y valoración de los costos de la acción política son similares entre los grupos, no siendo un factor decisivo en la determinación de participación en acciones colectivas. Similares resultados fueron encontrados anteriormente por Klandermans (1984) y Etxebarría, Páez y Valencia (1988b).

Tratamos igualmente de determinar si se encontraban diferencias entre las intenciones de comportamiento hacia un cambio social radical (socialismo) e independentista (independencia) (ambas escalas observaron una alta correlación $[\mathrm{r}=.80, \mathrm{p} \leqslant .0001]$ ). Esto nos llevó a concluir, por tanto, que las variables independientes consideradas tendrían un comportamiento similar, lo que quedó confirmado. Dada la similitud con los resultados de la tabla anterior no se presentarán estos últimos por ser redundantes (ver $\mathrm{Va}$ lencia 1987). Sin embargo, encontramos que al adoptar como Variable Dependiente la Intención de participar en acciones colectivas dirigidas a la consecución del socialismo, la clase social diferenció entre los tres grupos, siendo los sujetos de clase más baja los que manifestaron una intención de acción más «radical" para su consecución $(\mathrm{F}=4.63, \mathrm{p} \leqslant .0107)$. Por el contrario, no se encontraron diferencias significativas al tomar la intención de acciones colectivas cara a la consecución de la independencia $(\mathrm{F}=1.28$, $p \leqslant .2803$ ). Sin embargo, al considerar la pertenencia a la comunidad lingüística "euskaldún", si bien en ambos casos diferenció entre los tres grupos, fue en el caso de la independencia donde la diferencia fue más importante $(\mathrm{F}=9.66, \mathrm{p} \leqslant .0001$, frente a $\mathrm{F}=6.43, \mathrm{p} \leqslant .0020)$. Esto es lógico a la luz de las teorías de la categorización social (Tajfel, 1978, Turner y Brown 1978, Turner 1985), ya que ante la independencia como objeto de análisis la "identidad lingüística» ocupará una dimensión especialmente relevante de comparación intergrupal, mientras que en el socialismo, la dimensión clase social será más saliente.

A modo de resumen podemos apuntar que los resultados sugieren que hay factores Macro-ideológicos «objetivos" creadores de redes de responsabilidad y solidaridad grupal, que al igual que factores individuales y provenientes de las relaciones interindividuales, indican fuertes relaciones con la participación política no institucional.

En relación con el análisis de la influencia de las variables tomadas en cuenta a los 3 niveles, realizamos un análisis de regresión stepwise con introducción jerárquica de las variables correspondientes a cada nivel.

Encontramos (ver tabla XI) que la introducción sucesiva de las varia- 
TABLA XI

Introducción sucesiva de los diferentes niveles con npgtot

\begin{tabular}{|c|c|c|c|c|}
\hline & e & $\mathbf{R}$ & $r^{2}$ & $\begin{array}{c}\text { g.l. } \\
\text { (residuales) }\end{array}$ \\
\hline 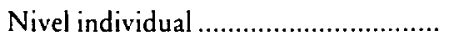 & 20 & .80 & .64 & 198 \\
\hline Nivel psicosocial ................................ & 16 & .81 & .66 & 194 \\
\hline Nivel macroideológico ............................ & 15 & .84 & .70 & 187 \\
\hline
\end{tabular}

bles de nivel psicosocial y macroideológico aumentan significativamente la varianza explicada y disminuyen el error, siendo las ratios de $F$ incluidas significativas. Realizamos también regresiones de cada uno de los niveles separadamente sobre la variable dependiente, encontrando que si bien es el nivel individual quien mayor porcentaje de varianza explica $(.64 \%$, $\mathrm{F}=39.38, \mathrm{~S}=.00$ ), los niveles psicosocial y macroideológico explican cada uno de ellos por separado entre un $43 \%(F=38.37, \mathrm{~S}=.0000)$ y $44 \%$ $(\mathrm{F}=22.79, \mathrm{~S}=.00)$.

Un último punto de nuestro trabajo constituía la hipótesis de un campo representacional diferenciado para los sujetos políticamente radicales. En otros términos, no sólo las variables discriminarian entre los diferentes niveles de participación, o predecerían la intención de conducta, sino que también estarían articuladas entre ellas, constituyendo una estructura representacional unificada.

Con el fin de verificar la hipótesis del campo representacional diferenciado de los sujetos radicales, en lugar de utilizar niveles de participación, definimos los sujetos en función del voto y realizamos un análisis de correspondencias (SPAD) con todas las variables utilizando sus puntuaciones terciles. Con el fin de contrarrestar los resultados del análisis de correspondencias, sometimos las variables tricotomizadas (excepto Sexo (SEX), Creencias Normativas Personales (CRN) y Militancia (MIL) a la prueba de $\mathrm{X}^{2}$ (ver tabla XII) con voto definido de la siguiente manera:

VOT1 $=$ RADICALES $(E M K$, AUZOLAN, HB), VOT2 = EUSKADIKO EZKERRA, VOT3 = PARTIDO COMUNISTA, VOT $4=$ PSOE, $\mathrm{VOT} 5=$ PNV y EA, VOT6 $=\mathrm{AP}, \mathrm{VOT} 7=\mathrm{CDS}$, VOT $8=$ OTROS, VOT9 $=$ NOVOTO .

Posteriormente sometimos los datos a un análisis de correspondencias múltiples (SPAD). El análisis creó 6 factores que explicaban el $34 \%$ de la varianza. El primer factor explicó un 14,19\%, el segundo 5,47\%.

El primer factor en su polo negativo estaba definido por las siguientes contribuciones: baja participación no institucional (PMS1), baja valoración del motivo colectivo (MOT1), y baja privación relativa (PRI1), visión del mundo justa (MUD1), identidad social baja (GRP1). El polo positivo, en cambio estaba definido por alta participación tanto institucional como no institucional (PMS3, PMI3, PIS3, PII3), como alta privación (PRI3), alta anomia política (ANO3), visión del mundo injusta (MUD3).

El segundo factor tanto en su polo positivo como negativo estaba definido por la contribución de las categorías medias de participación diferenciándose en la experiencia anterior. 


\section{4}

TABIA XII

\begin{tabular}{|c|c|c|c|c|}
\hline & Variable & $\mathrm{X}^{2}$ & gl. & $\mathbf{p}$ \\
\hline SEX & $(\operatorname{sexo})$. & 5.05 & 6 & $.53^{*}$ \\
\hline EDA & 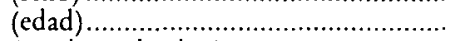 & 22.92 & 12 & .0021 \\
\hline MOT & (motivo colectivo) & 45.28 & 12 & .0000 \\
\hline NOR & (norma subjetiva) & 5.82 & 12 & $.92 *$ \\
\hline PAR & (importancia de la propia participa- & & & \\
\hline & ción) & 27.20 & 12 & .0073 \\
\hline MOV & $\begin{array}{l}\text { (actitud hacia movimientos colecti- } \\
\text { vos) }\end{array}$ & 51.37 & 12 & .0000 \\
\hline CLA & (clase social) ……………………..... & 20.83 & 12 & $.28^{*}$ \\
\hline PRI & (privación grupal) & 48.55 & 12 & .0000 \\
\hline MUD & (mundo injusto) & 32.58 & 12 & .0011 \\
\hline ATR & 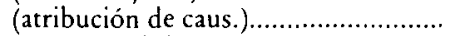 & 26.34 & 12 & .0000 \\
\hline ANO & 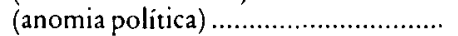 & 90.29 & 12 & .0000 \\
\hline SUJ & 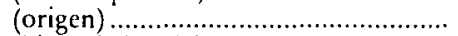 & 46.77 & 30 & .0264 \\
\hline GRP & 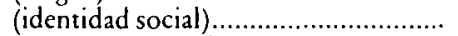 & 37.01 & 12 & .0002 \\
\hline MIL & 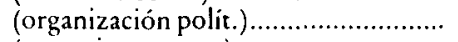 & 35.03 & 6 & .0000 \\
\hline CRE & 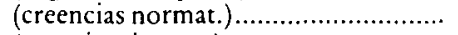 & 26.90 & 6 & .0002 \\
\hline EXP & (experiencia ante.) & 54.89 & 12 & .0000 \\
\hline PMS & 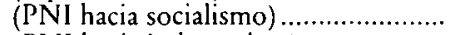 & 48.97 & 12 & .0000 \\
\hline PMI & 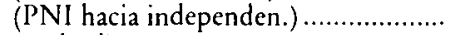 & 36.85 & 12 & .0002 \\
\hline UMB & (umbral) & 18.96 & 12 & $.0892 * \%$ \\
\hline ESK & (euskera) & 25.74 & 12 & .0117 \\
\hline $\cos$ & (costos percibidos) & 8.60 & 6 & $.3774 \%$ \\
\hline VAC & (valor de costos) .................................. & 7.39 & 6 & $.0832 \%$ \\
\hline PIS & (PPI hacia socialismo) & 38.82 & 12 & .0000 \\
\hline PII & (PPI hacia independen.) .......................... & 39.63 & 12 & .0000 \\
\hline ACS & (radicalismo social) $\ldots \ldots \ldots \ldots \ldots \ldots \ldots \ldots$ & 43.48 & 12 & .0000 \\
\hline IDB & 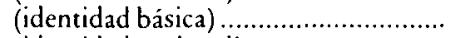 & 86.48 & 12 & .0000 \\
\hline IDN & 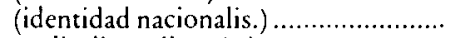 & 96.25 & 12 & .0000 \\
\hline IDL & 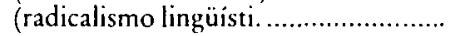 & 81.30 & 12 & .0000 \\
\hline
\end{tabular}

En la figura 5 se observa la proyección de los puntos de las contribuciones de las diferentes variables sobre los ejes 1 y 2.

Los resultados indican que encontramos un núcleo representacional de significado diferenciado de los radicales vascos, que incluye elementos estructurales ideológicos, recursos intergrupales articuladores, y recursos más individuales relacionados con elementos comportamentales tanto institucionales como no institucionales. Con el fin de corregir los resultados del análisis de correspondencias mediante el procedimiento Tri-Dos, se calculó la correlación entre las diversas variables (ver Valencia, 1987).

Encontramos que los votantes RADICALES (VOT1) observan una actitud radical ante el cambio social (ACT3), una visión distante del sistema político oficial (ANO3), una fuerte identidad nacionalista vasca (IDN4), alto radicalismo lingüístico (IDL4), una identidad política de extrema izquierda (IDB5), realizan atribuciones de causalidad externa (ATR3), una fuerte valoración de la independencia y del socialismo (MOT3), una creencia normativa personal de que deben participar en movimientos colectivos (CRN2), una actitud positiva hacia los movimientos colectivos (MOV3), una alta intención de participación no institucional tanto de cara a la consecución de la independencia como del socialismo (PMS3, PMI3), así como una alta intención de participación institucional (PII3 PIS3), están organizados políticamente (MIL2), un umbral de participación bajo (UMB1), así como una experiencia anterior alta (EXP3). Igualmente, aunque con corre- 


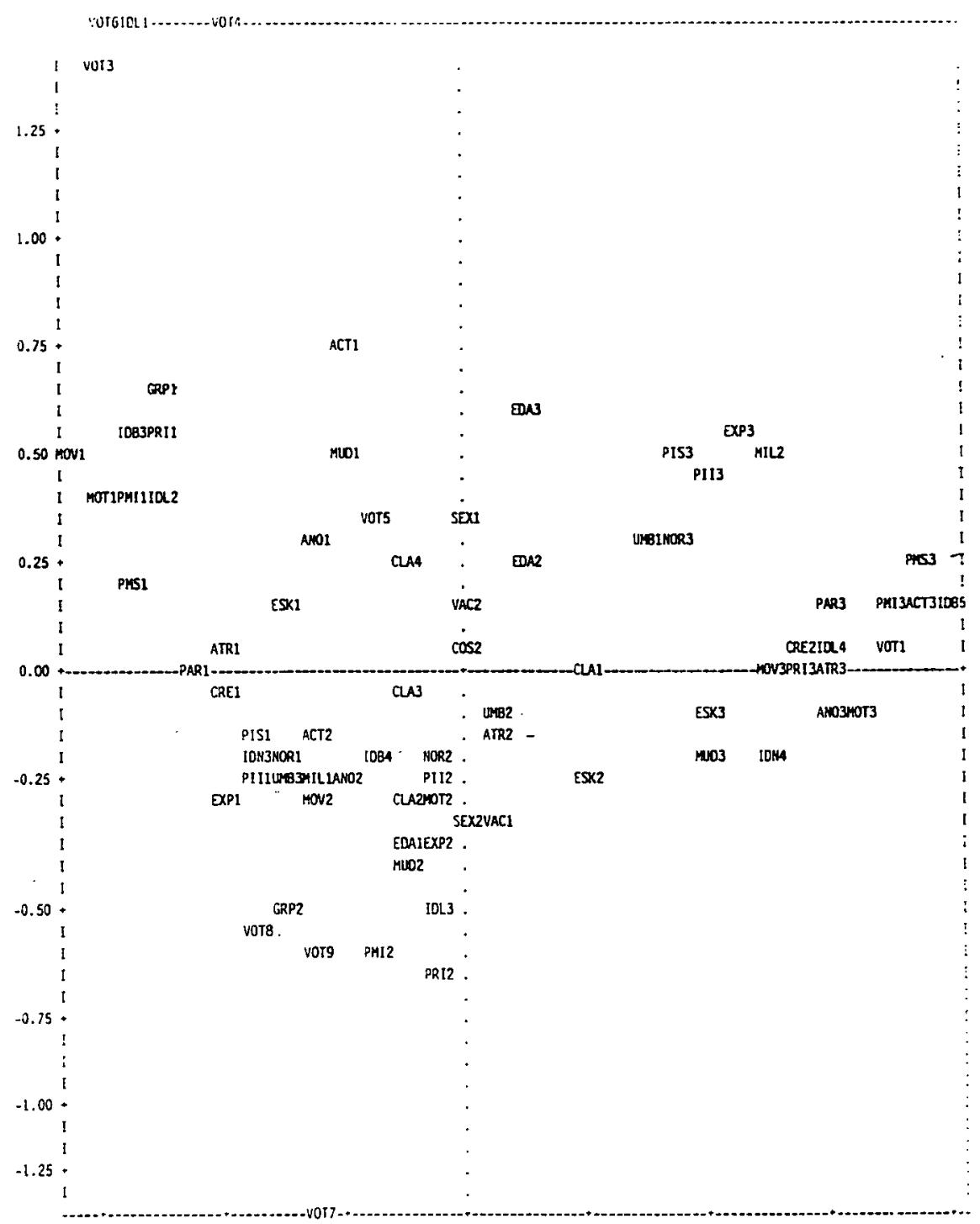

laciones más bajas, los radicales vascos observan una visión del mundo político injusto (MUD3), una alta privación grupal (PRI3), una identidad social vasca (GRP3), tienen una alta competencia de participación (PAR3). Finalmente, con correlaciones bajas, pero cercanos en el campo representacional, encontramos la clase social baja (CLA1), y un conocimiento del euskera alto (ESK3).

A modo de conclusión los resultados sugieren que se confirma una representación social de la participación política no institucional diferenciada por parte de los sujetos radicales, apareciendo las variables antes definidas en los diversos niveles de análisis macro, microsocial y psicológico asociados a niveles elevados de participación no institucional. Además, los suje- 
tos implicados en acciones radicales no institucionales aparecen claramente diferenciados del resto, que aparecen compartiendo un núcleo poco diferenciado de características.

Estos resultados hacen incidencia en la Teoria formulada por Tajfel (1978) y Turner $(1978,1987)$ sobre la Identidad Social. Recordemos que según dicha formulación, cuando un grupo en la comparación social está en una situación de desventaja o inferioridad (en nuestro caso puede traducirse en acceso al poder y posibilidades de desarrollo de la propia identidad), para que dicha situación conduzca a un Movimiento por el Cambio Social deben ocurrir diversos elementos: la dimensión de comparación desfavorable debe ser percibida como ilegitima, existiendo simultáneamente dificultades para la movilidad social. En nuestro caso, las variables consideradas (Privación Grupal, e Identidades Social, Nacionalista y Básica a nivel intergrupal, y atribución de Causalidad y percepción injusta del mundo a niveles más individuales) reflejan la percepción de injusticia percibida por parte de los grupos nacionalistas radicales (HB, AUZOLAN, EMK). Así mismo, como lo refleja claramente el análisis de correspondencias, existe una clara definición del endo y exo grupo de función de factores lingüísticos y étnicos etc... Esta situación explicaría en una parte importante la disposición de dichos grupos para movilizarse por el cambio social, así como fenómenos de discriminación grupal (favoritismo intragrupal y discriminación hacia los exogrupos característicos de la situación social analizada) (Reicher, 1987, Moscovici y Pacheler, 1978, Turner y Brown, 1978, Van Knippenberg, 1978, McKie 1986, Richardson \& Cialdini, 1981).

\section{DISCUSION}

A la vista de los resultados obtenidos se pueden alcanzar conclusiones de dos tipos diferentes. En primer lugar, relativas a los modelos de los que partimos para formular nuestras hipótesis. Son éstos el de Actitudes, el de Klandermans, el de Olson y el Psicosocial. En segundo lugar, relativas a las hipótesis en sí mismas consideradas, es decir, a la utilidad de las actitudes y las normas subjetivas, a la necesidad de considerar la existencia de recursos psicológicos, ideológicos y de factores estructurales, a la importancia de aceptar una racionalidad de corte culturalista y, finalmente, a la conveniencia de tomar en cuenta explicaciones no exclusivamente causales.

\section{Modelo de Actitudes}

Hemos visto cómo los dos factores tradicionales del Modelo (Actitud y Norma Subjetiva), son elementos válidos para la explicación de la Participación Política No Institucional. Los resultados de este trabajo sugieren que con medidas correctas se puede superar la inconsistencia encontrada entre las actitudes de los sujetos y su intención comportamental (o su comportamiento posterior). Hemos visto también cómo la introducción de la Experiencia Anterior en el modelo, aumenta la varianza explicada de éste, debido al papel que juega en la intención comportamental.

Encontramos también el rol de la Creencia Normativa Personal, como elemento normativo "moral" de los actores políticos radicales ("yo debo participar en comportamientos colectivos"). Hemos visto que su introduc- 
ción en el modelo aumenta la varianza explicada de éste, pudiendo jugar un papel de sustituto de la Norma Subjetiva. Este hecho nos puede hacer reflexionar con respecto a la PPNI, en el sentido de que fines moralmente relevantes para los actores sociales como pueden ser el logro de una sociedad socialista e independiente, junto con medios «legitimados" para el logro de aquéllos, sean factores a tener en cuenta, incluso a veces más que factores puramente situacionales. Por otra parte, hemos visto también, por medio de un diagrama de senderos, como puede cumplir un papel mediador entre la actitud y la norma social, y la intención de participación, no teniendo la experiencia anterior un efecto, sino en la intención de participación, en el sentido que la gente recuerda su pasado a la hora de tomar una decisión comportamental. Esta problemática, sin embargo, no está resuelta, y sigue abierta, tanto a nivel teórico como empírico, pues si bien en este trabajo no hay evidencia, también es cierto que la gente a veces revisa su pasado para hacerlo consistente con su Intención Ideal Comportamental, con su Creencia Normativa Personal. Quizá, en este sentido, el Modelo de Proceso de Fazio y cols., con su análisis de la «accesibilidad de la actitud", y con la importancia dada a la formación de la actitud mediante contacto directo con el objeto actitudinal, pueda ayudarnos en el futuro a dar luz sobre el problema.

\section{Modelo de Klandermans}

Como el de Actitudes, se desarrolla a partir de los Modelos de Utilidad y Valor Esperado. Hemos visto cómo es el Motivo Colectivo, lo que más peso tiene en el PPNI. El Motivo social (operacionalizado, siguiendo a Klandermans, de manera similar a la Norma Subjetiva del modelo anterior) aumenta la varianza explicada, produciendo entre ambos factores una varianza explicada mayor que el modelo anterior, y no es significativa la introducción del Motivo de Recompensa.

Debido a que el Motivo de Recompensa no tuvo influencia en la PPNI, y el Motivo Social estaba igualmente operacionalizado que el anterior, podemos considerar que el elemento que produjo el mayor monto de explicación al Modelo de Klandermans fue el Motivo Colectivo, en especial su factor Expectativa de participación exitosa, a diferencia de la Instrumentalidad del modelo de Fishbein. El factor «Expectativa de participación exitosa", incluye los aspectos de solidaridad grupal de cara a la racionalidad colectiva, así como el elemento tradicionalmente relacionado con la PPNI de Sentimiento de Competencia.

\section{Modelo de Olson}

De cara al dilema de la Racionalidad Individual-Colectiva, en contraposición a la hipótesis de Olson de que los beneficios selectivos «exclusivos y separados" son necesarios para un comportamiento colectivo, podemos observar que la PPNI no será un subproducto de aquéllos. El Valor del Bien Colectivo (valor de la Independencia y del Socialismo), junto con los elementos facilitadores de sentimientos de solidaridad grupal de la «Expectativa de participación exitosa", son los que obtienen mayor peso facilitador de la participación, así como el Motivo Social.

En este mismo sentido, se ve relevante la introducción del Umbral de 
Participación, como elemento de Solidaridad Grupal facilitador de la PPNI, haciendo que el "Número esperado de participantes" no tenga incidencia en la Participación, quizá por la problemática no resuelta todavía, y gráficamente descrita por P. Oliver: «si tú no lo haces, nadie más lo hará».

\section{Modelo Psicosocial}

La integración sucesiva de los niveles intergrupal y macroideológico con el nivel individual, aumenta la varianza explicada de la PPNI. Este modelo fue el que obtuvo mayor varianza explicada de la PPNI. Este análisis aduce en el sentido de los planteamientos metateóricos según los cuales los fenómenos sociales, son "abiertos" y complejos, estando estratificados a diferentes niveles. Nosotros, basándonos en las aportaciones psicosociales de W. Doise, consideramos que de cara a la explicación del comportamiento colectivo como la PPNI, no podemos asumir solamente elementos de corte individual, ni puramente colectivos, sino que será la integración de ambos por medio del nivel intergrupal, la que ayude a dar, desde la Psicología Social, una respuesta a la PPNI. Recordemos que como dice S. Moscovici la Psicología Social es la ciencia del conflicto entre el individuo y la sociedad.

En este sentido, si bien no integrado en el Modelo Psicosocial por falta de medidas adecuadas, el papel integrador que realiza la Representación Social puede ser relevante. En el campo representacional, el esquema temático figurativo juega un papel estructurante del resto de los contenidos, dando estabilidad a la Representación Social. Así, se observa que la estructura del campo representacional de los participantes radicales vascos, compuesta por alta anomia política, cambio social radical, privación grupal alta, visión del mundo injusto, atribución de causalidad externa, así como por experiencia anterior e intención de participación radical altas, confirman los resultados de Villarreal (1987), que encontró estos factores asociados a una Representación Social (inferida por asociación libre de palabras) de carácter fuertemente político, con elementos de intensidad emocional, y asociados a intenciones de comportamiento radical.

Por lo que respecta a las hipótesis formuladas y aun siendo conscientes de las limitaciones de este trabajo (muestra -clase social, y edad-, instrumentos - bajas fiabilidades en las escalas de mundo justo-, metodológicas - utilización de regresión múltiple en lugar de modelos de ecuaciones estructurales, problemas de multicolinealidad-, excesivo número de variables, medición de variables dependientes como Intención de participación o Potencial de participación, en lugar de comportamiento real), consideramos que podemos llegar a las conclusiones siguientes:

1. En la explicación de la Participación Política No Institucional, el empleo de las Actitudes y las Normas Subjetivas nos pueden ayudar en la tarea, pero no son suficientes.

2. Al tomar en consideración un Modelo «Lógico Racional» explicativo de la Participación Política No Institucional, más que la premisa de que son los Incentivos Selectivos los que hacen participar al «beneficiario franco», juzgamos que son el Valor del Bien Colectivo (la Independencia y el Socialismo), así como la Expectativa de Participación Positiva, y la asunción de RECURSOS PSICOLOGICOS como la Atribución de Causalidad Externa, una visión 
del Mundo Injusto, así como el Umbral de Participación y la Experiencia Anterior, los elementos facilitadores de la Participación a un nivel más individual. Igualmente, será necesaria la asunción de recursos facilitadores de SENTIMIENTOS DE SOLIDARIDAD GRUPAL como la Identidad Social Vasca, la Identidad Nacionalista, la Identidad Básica, la Privación Grupal, y las Representaciones Sociales. Finalmente, será necesaria también la asunción de RECURSOS MAS IDEOLOGICOS como la Anomia Política, el Radicalismo Lingüístico, así como FACTORES ESTRUCTURALES como el Origen, la Edad, la Clase Social y la Lengua.

En este sentido, y pensando en posteriores profundizaciones de los "Beneficios Selectivos», sería interesante hacer hincapié en variables de «ingreso písquico" como el "gusto por ir a manifestaciones", "conocer a nuevos amigos", etc., o incentivos negativos como el ser etiquetado como "rojo", "separatista», "violento», etc...

3. Refiriéndonos a la Racionalidad de la Acción Colectiva, cabe apuntar que una racionalidad puramente utilitarista, que busque maximizar sus intereses, no será suficiente. Será necesaria también una recionalidad de corte más culturalista, que tenga en cuenta las «buenas razones» de tipo sociocultural, como las actitudes, identidades sociales, representaciones sociales, aspectos ideológicos y culturales, si bien no podrá ser olvidado el papel "constreñidor" de las estructuras sociales.

4. Finalmente, en cuanto a la Explicación de la PPNI en particular, y de los comportamientos colectivos en general, apuntar que si bien la explicación causal ocupa un lugar relevante, la existencia de la mente y la intencionalidad en los actores colectivos, nos lleva a sugerir que las Razones pueden ser Causas. El conjunto de creencias y opiniones (por utilizar términos más descritivos) que los sujetos radicales tenían sobre el mundo social, estaban asociadas, y permitían "predecir" sus intenciones de conductas. En este sentido, las razones de los radicales vascos eran "causas" potenciales de su conducta. Estas razones tenían además una estructura de significado -según vimos en el análisis de correspondencias-articuladora de elementos afectivos, cognitivos y comportamentales.

\section{Bibliografía}

Adorno, T. W., et al. (1950). The Autoritarian Personality, NY: Harper.

AJZEN, I., y FISHBEIN, M. (1973). "Atritudinal and normative variables as predictors of specific behaviors". JPS Psy., 27: 41-57.

AJZEN, I., y FishBEIN, M. (1980). Understanding Attitudes and Predictiong Social Behavior. NJ: Prentice Hall.

Almond, G. A., y Verba, S. (1965). The Civic Culture: Political attitudes and democracy in five nations, Boston: Little Brown.

Almond, G. A., y Verba, S. (1980). The Civic Culture Revisated, Boston: Little Brown.

ALZATE, R. (1980). La relación actitud conducta: un estudio aplicado a la conciencia nacional. Tesis Doctoral no Publicada. Univ. País Vasco.

ARENDT, H. (1972). Le systeme totalitaire. France: Seuil.

BANDURA, A. (1973). Agression: A social learning analysis. NJ: Prentice Hall.

Barnes, S. H., y KaAse, M. (1979). Political Action. Beverly Hills: Sage. 
Bentler, P. M., y SPECharT, G. (1979). "Models of attitude-behavior relations". Psy. Rev., 86: $452-464$.

BENTLER, P. M., y SPECHART, G. (1981). «Attitudes "cause" behaviours: a structural analysis». JPS Psy., 40: 226-238.

BHASKAR, R. (1978a). A Realist Theory of Science, Brighton, Sussex: Harvester.

BHASKAR, R. A. (1983). "Emergence, explanation and emancipation» in Secord, P. F. (ed.) Explaining human behavior: conciousness, buman action and social structure. Beverly Hills: Sage.

BowMAN, L. H., y Fishbein, M. (1978). "Understanding public reaction to energy proposals: An application to the Fishbein Model». J. Appl. S. Psy., 8: 319-340.

BUDD, R. J.; NORTH, D., y SPENCER, C. P. (1984a). "Understanding seat belt use: A test of Bentler and Speckart's extension of the theory of reasoned action". Eur. JS Psy., 14:69-78.

BUDD, R. J., y SPENCER, C. P. (1984b). "Predicting undergraduates» intentions to drink. J. Stud. Alcohol, 45: 179-183.

BUDD, R. J., y SPENCER, C. P. (1985). «Exploring the role of personal normative beliefs in the theory of reasoned action: The problem of discriminating between alternative path models». Eur. JS Psy., 15: 299-313.

DAviES, J. C. (1962). "Toward a theory of revolution". Am. Soc. Rev., 27: 5-19.

Dawes, R. M. (1980). «Social Dilemmas». Ann. Rev. Psy., 31: 169-193.

Della PoRTa, D., y MatTina, L. (1986). “Ciclos políticos y movilización étnica: el caso vasCo". REIS, 35: 123-148.

DOISE, W. (1976). L'articulation psychosociologique et les relations entre groupes. Bruselas: De Boek.

DOISE, W. (1979). Psicología Social y Relaciones entre Grupos (vol. 1) Barna: Rol.

DOISE, W. (1980). «Levels of explanation in the European Journal of Social Psychology». Eur. JS Psy., 10: 213-231.

DOISE, W. (1983). «Tensiones y Explicaciones en Psicología Social Experimental». Revista Mexicana de Sociologia, 45: 659-685.

DuBE, L., y GUIMOND, S. (1986). «Relative deprivation and social protest: the personal-group issue», en Olson, J.; Herman, P., y Zanna, M. (eds.) Relative Deprivation and Social Comparison. NJ: Erlbaum: 201-216.

DumONT, P. (1982). Facteurs Psychosociaux et Idèologiques du militantisme pacifiste. Tesis Doctoral no publicada. Facultad de Psicología. UC Lovaina.

ELSTER, J. (1984). «Funcionalismo y T." de juegos. Alegato en favor del individualismo metodológicom. Zona Abierta, 33: 21-62.

Etxebarría, A.; Paez, D., y Valencia, J. F. (1988a). "Testing Ajzen and Fishbein' Attitudes Model». EJS Psy., 18: 181-190.

Etxebarkía, A.; Paez, D., y Valencia, J. F. (1988b). "Toma de decisión estratégica y Participación en Comportamientos Colectivos: el caso del nacionalismo vasco». Revista Vasca de Psicología, 4: 26-37.

FishbeIn, M., y AJZEN, I. (1975). Belief, Attitude, Intention, and Behavior. N.Y.: AddisonWesley.

FISHBEIN, M., y COOMBS, F. S. (1974). "Basis for decision: an attitudinal analysis of voting behavior». J. Appl. S. Psy., 4: 95-124.

FISHBEIN, M.; ThOMAS, K., y JACCARD, J. J. (1976). «Voting behavior in Britain: An attitudinal analysis". Occasional Papers in Survey Research, 7. Survey Unit. Londres.

Freud, S. (1976). Psicología de las masas y análisis del Yo. Madrid: Biblioteca Nueva. (Original 1923).

Gamson, W. (1975). The Strategy of Social Protest. Illinois: Dorsey.

GAMSON, W.; FIREMAN, B., y RYTINA, S. (1982). Encounters with Unjust Authority. Illinois: Dorsey.

Garmendia, J. A., et al. (1982). Abertzales y Vascos. Madrid: Akal.

GILES, H.; BOURISH, R., y. TAILOR, D. M. «Towards a Theory of Language in Ethnic Group Relations" en Giles, H. (ed.): Language, Ethnicity, y Intergroup Relations. Londres: Academic Press.

GILES, H., y JOHNSON, P. (1984). "The role of lenguage in ethnic group relations. in Turner, J., y Giles, H. (eds.): Intergroup Bebaviour. Londres: Blackwell.

GORUSCH, R. L., y ORTBERG, J. (1983). "Moral obligation and attitudes". JPS Psy., 44: 1025-1028.

GranovetTER, M. (1978). "Threshold models of collective behavior". Am. J. Soc., 83: $.1420-1443$.

GROH, D. (1986). «Collective behavior from the 17th to the 20th century: change of phenomena, change of perception, or no change at all? Some preliminary reflections", en Graumann, C. y Moscovici, S. (eds.): Changing conceptions of Crowd Mind and Behavior. NY: Springer.

GuimOND, S., y DubÉ-Simard, L. (1983). «Relative deprivation theory and Quebec nationalist movement: the cognition-emotion distinction and the personal-group deprivation issue». JPS Psy., 44: 526-535. 
GUNTER, B., y WOBER, M. (1983). "Television viewing and public trust". BRJS Psy., 22. 174-176.

GURNEY, D. N., y TiERnER, K. T. (1982). "Relative deprivation and social movements». Soc. Quar., 22: 33-47.

GURR, T. R. (1970). Why Men Rebel. NJ: Princeton Univ. Press.

HARIIN, R. (1982). Rational Man and Irrational Society. Beverly Hills: Sage.

HARDIN, R. (1982). Collective Action. Baltimore: John's Hopkins.

HECHTER, M. (1975). Internal colonialism: the celtic fringe in British National Development. Berkeley: Univ. Cal. Press.

Hermann, M. G. (1986). Political Psychology. Londres: Jossey-Bass.

HyLAND, M. E., y DANN, P. L. (1987). "Exploratory factor analysis of the Just World Scale using British undergraduates.. Br. JS Psy., 26: 76-77.

JaSPARS, J., y HEWSTONE, M. (1984). "La theorie de l'attribution", en Moscovici, S. (ed.): Psychologie Sociale. Paris: PUF.

JENKINS, J. C. (1982). "The transformation of a constituency into a movement", en Freeman, J. (ed.). The Social Movements of the 1960's and 1970's. NY: Longmans.

JENKINS, J. C. (1983). "Resource mobilization theory and the study of social movements". Ann. Rev. Soc., 9: 527-553.

KAASE, M., y MARSH, A. (1979a). "Distribution of political action», en Barnes, S. H. y Kaase, M. (eds.). Political Action. Beverly Hills: Sage.

KAASE, M., y MARSH, A. (1979b). "Political action repertory: changes over time and a new tipology", en Barnes, S. H., y Kaase, M. (eds.) Political Action. Beverly Hills: Sage.

KINDER, D. R., y SEARS, D. O. (1985). "Public Opinion and Political Action», en Lindsey, E., y Aronson, G. (eds.): Handbook of Social Psychology (Vol. 2). NY: Rendon House.

KLANDERMANS, B. (1984). "Movilization and participation: social psychological expansions of resource movilization theory». Am. Soc. Rev., 49: 583-600.

KLANDERMANS, B. (1985). "Individuals and collective action». Am. Soc. Rev., 50: 860-861.

Klandermans, B., y Oegema, D. (1987). "Potentials, Networks, motivations and Barriers». Am. Soc: Rev., 52: 519-531.

KORNhauser, W. (1978). "La société de masses», en Birnbaum, P., y Chazel, F. (eds.). Sociologie Politique. Paris: Colin.

Kourevatis, y Dobratz, R. (1982). "Political power and Conventional political participation.. Am. Soc. Rev. 8: 269-317.

LAMBERT, W. E. (1979). "Language as a Factor in Intergroup Relations", en Giles, H., y St. Clair, R. (eds.): Language and Social Psychology. Londres: Blackwell.

LE BON, G. (1961). Psicologia de las Multitudes. La Habana: Cuba (original 1895).

LEDERER, G. (1986). "Protest movements as a form of political action", en Herman, M. G. (ed.): Political Psychology. Londres: Jossey-Bass.

LERNER, M. J., (1970). "The desire for justice and reactions to victims", en Macaulay, J. y Berkowitz, L. (eds.): Altruism and belping behavior. NY: Ac. Press.

LERNER, M. J., y MILLER, D. T. (1978). "Just world research and the attribution process". Psychol. Bull., 85: 1030-1051.

LINZ, J., et al. (1981). Informe Sociológico sobre el cambio político en España. Madrid: Foessa.

MANICAS, P. T., y SECORD, P. F. (1983). "Implications for psychology of the new philosophy of science». American Psychologist., 35: 399-413.

Maravall, J. M. (1978). Dictadura y Disentimiento Politico. Madrid: Alfaguara.

MATTEI, F. (1986). "Olson e la "Legge Ferrea" della partecipazione». Rivista Italiana Di Scienza Politica, 16: 123-148.

McAdam, D. (1982). Political Process and the Development of Black Insurgency. Chicago: Chicago Univ. Press.

McAdAm, D.; McCARTHY, J., y ZaLD, M. (1988). «Social Movements", en Smelser (ed.): Handbook of Sociology. Beverly Hills: Sage.

McCarThy, J., y ZaLD, M. N. (1977). "Resource movilization and social movements". Am. J. Sociol., 82: 1212-1241.

McKIE, D. M. (1986). "Social Identification effects in group polarization». J. P. S. Psy., 50: 720-728.

Milbrath, L. W. (1981), "Political Participation», en Long, S. L. (ed.): The Handbook of Political Behavior. NY: Plenum Press.

MOE, T. (1980). "A calculus of group membership". Am. J. Pol. Science, 24: 1-29.

Moscovici, S. (1981). L'áge des Foules. París: Fayard.

Moscovicl, S. (1986). "The discovery of the masses", en Graumann, F., y Moscovici, S. (eds.): Changing Conceptions of Crowd Mind and Behavior. NY: Springer.

MosCovici, S., y PACHELliR, G. (1978). «Social comparison and social recognition: Two complementary processes of identification", en Tajfel, H. (ed.): Differentiation between social groups. Londres: Academic Press.

Muil.ter, E. N., (1979). Aggresive Political Participation. NY: Princenton Univ. Press.

MUi.t.eR, E. N. (1982). "An explanatory model for differring types of participation». European Journal of Political Research, 10:1-16. 
Muller, E. N., y OpP, K. D. (1986). «Rational choice and rebellious collective action». Am. Pol. Sc. Rev., 80: $471-487$.

NEwCOMB, T. (1951). «Social psychological Theory. Integrating individual and social approaches", en Roherer, J., et al. (eds.): Social Psychology at the crossroads. NY: Harper.

NIE, N. H., y VERBA, S. (1975). "Political participation", en Greenstein, F., y Polsby, M (eds.): Handbook of Political Science. USA: Addison-Wesley (vol. 4).

NiELSEN, F. (1980). "The flemish movement in Belgium after World War II: a dynamic analisis". Am. Soc. Rev., 45: 76-94.

NifLSEN, F. (1985). "Toward a theory of ethnic solidarity in modern societies". Am. Soc. Rev., 50: 133-149.

Oberschall, A. (1973). Social Conflict and Social Movements. NJ: Prentice Hall.

OLIVIER, P., et al. (1984). «If you don't do it, nobodv else will: active and token contributors to local collective action". Am. J. Rev., 49: 601-610.

Olson, M. (1979). La Logique de L'Action Collective. París: PUF (original 1965).

Olson, M. (1986). Auge y decadencia de las Naciones. Madrid: Ariel.

PÁEZ, D. (1982). Psichosociologie des Comportements Collectifs et des Movements Sociaux. Tesis doctoral no publicada. Depto. Ps. Social, Universidad de Lovaina.

PÁEZ, D. y ECHEBARRIA, A. (1986). "Participación política institucional y extra-institucional", Servicio Central de Publicaciones (ed.): Psicosociologia del Adolescente Vasco. Bilbao: Lan Osasunketa eta Gizarte Saila.

PORTES, A. (1971). «Political primitivism, differential socialization, and lower-class frustration as the cause of leftist radicalism». Am. Soc. Rev.

ReEs, G. (1985). "Introduction: class, locality and Ideology", en Rees, G., et al. (eds.): Political action and social Identity. Londres: McMillan.

REICHER, S. (1987). “Crowd behavior as social action", en Turner, J. C. (ed.): Rediscovering the social group. Oxford: Blackwell.

Ross, J. A. (1979). "Language and the mobilization of ethnic identity", en Giles, H., y SaintJacques; B. (eds.): Language and Ethnic Relations. Oxford: Pergamon.

RubiN, Z., y Peplau, A. (1975). «Who believes in a Just World?" J. of S. Issues, 3l: 65-89.

SABucedo, J. M. (1984). «Psicología y participación política». Boletín de Psicologia, 5: 61-77.

SABUCEDO, J. N., y SOBRAL, J. (1986). "Participación política y conducta de voto". Papeles del Colegio de Psicólogos, 25: 15-22.

SCHRAGER, L. S. (1985). "Private attitudes and colective action». Am. Soc. Rev., 50: 858-860.

SCHWARTZ, M. (1976). Radical Protest and Social Structure. NY: Academic Press.

SCHWARTZ, S. H. (1978). "Temporal instability as a moderator of the attitude-behavior relationship». J. P. S. Psy., 36: 715-724.

Sears, D. O. (1987). "Political psychology». Ann. Rev. Psy., 38: 229-255.

SECORD, P. F. (1982). «Integrating the Personal and the Social», en Secord, P. F. (ed.): Explaining Social Behavior. Beverly Hills: Sage.

SECORD, P.F. (1986). "Explanations in the social sciences and in life situations", en Fiske, D. W., y Shweder, R. A. (eds.): Metatheory in Social Science: Pluralism and Subjectivities. Chicago: Chic. Univ. Press.

SelikTAR, O. (1986). "Identifying a society's belief systems", en Herman, M. G. (ed.): Political Psychology. Londres: Jossey-Bass.

SILVER, J. (1974), "Political revolution and repression: an economic approach". Public Choice, $17:$ : 677-682.

SMELSER, N. J. (1963). Theory of Collective Behavior. NY: Free Press.

SNOw, D. A.; ZURCKER, L. A., y EKLAND-OlsON, S. (1980). «Social networks and social movements". Am. Soc. Rev., 45: 787-801.

SNOw, D. A.; RoCHFORD, E. B., et al. (1986). «Frame Alignment processes, micromobilization, and movement participation». Am. Soc. Rev., 51: 464-481.

TABACHNICK, B. G., y FIDELL, L. S. (1983). Using Multivariate Statistics. NY: Harper.

TAJFEL, H. (1978). "Differentiation between social groups: studies in the social psychology of intergroup relations", en Tajfel, H. (ed.): European Monog. in Soc. Psyc. (n." 14). Londres: Academic Press.

TAjFel, H. (1984). The Social Dimension. Cambridge: Cambridge Univ. Press.

TAJFEL, H., y TURNER, J. C. (1979). "An integrative theory of intergroup conflict", en Austin, W. G., y Worchel, S. (eds.): Social Psychology of Integroup Relations. Londres: Cole.

Tilly, C. (1978). From Movilization to Revolution. Reading MA: Addison-Wesley.

TUlLOCK, G. (1971). "The paradox of revolution». Public Choice, 11: 89-99.

TURNER, J. C. (1985). "Social categorization and the self-concept», en Advances in Group Processes, 2: 77-121.

TURNER, J. C. (1987). Rediscovering the social group. A self Categorization Model. Oxford: Blackwell.

TURNER, J. C., y BROWN, R. (1978). «Social status, cognitives and intergroup relations", en Tajfel, H. (ed.): Differentiation between Social Groups. Londres: Academic Press.

VAN KNIPPENBERG, A. (1978). "Status differences, comparative relevance and integroup differentiation", en Tajfel, H. (ed.): Differentiation between Social Groups. Londres: Academic Press. 
Vali:NCIA, J. I., (1987). Racionalidad Individaal y Colectiva de la Participacion Politica No Institucional. Tesis no publicada. Depto. Ps. Soc. País Vasco.

VAl.ENCIA, J. F. y VILLARRLIAL., M. (1978). "Representaciones sociales: motivacion y Participación Politica No Institucionalm. Comunicación presentada en el l Congreso Hispano Luso de Psi. Social. Tomar.

Vit.l.ARrEal., M. "Representaciones Sociales de la Identidad Social y Factores Psicosociales asociados a la participación en Movimientos sociales Nacionalistas». Tesis No Publicada. Depto. Psi. Social. Univ. Pais Vasio.

WetsBY, J. (1976): The Clouded Vision. PA: Bucknell Univ. Press.

WILSON, J. Q. (1973): Political Organizations. NY': Basic.

WILLIAMS, C. H. (1979). "An ecological and behavioural analysis of ethnolinguistic change in Wales", en Giles, H., y Saint-Jaques, (eds.): Language and Ethnic Relations. Oxford: Pergamon.

ZURCHER, L. A., y SNOw, D. A. (1981). "Collective behaviour: social movements", en Rosenberg, M. y Turner, R. (eds.): Social Psychology, Sociological Perspectives. NY: Basic. 